\title{
Correlation between antimullerian hormone level before and after laparoscopic ovarian autologous PRP injections
}

\author{
Original MohammedAbdelgafoor Abdelgadir Salih, Mohammed Omer Mohammed Hussein, \\ Article Azhar Tag Aldin, Mosab Nouraldein Mohammed Hamad*, BadrEldin KaramAlla \\ Eljack, Hiba Osman Seid Ahmed Mohammed, Rayan Khalid Mohammed Ahmed
}

Banoon Fertility Center, Khartoum,Sudan

\begin{abstract}
Aim: Randomized clinical trial aimed to know the possible correlation between laparoscopic ovarian autologous PRP injection and AMH level.

Patients and Methods: Fifty-one Sudanese subfertile women attended to Banoon Fertility Center, Khartoum, Sudan, during the period from January 2018- June 2019 selected randomly and exposed to laparoscopic ovarian autologous PRP injection. AMH level of every participant measured before and after 3 months of administration of PRP to the ovaries.

Results and Conclusion: We measured size of correlation between AMH level pre and post exposure of ovaries to PRP, AMH level increased in 33.3\% (17) of the participants after PRP injection. Size of correlation $=0.64$ which represent moderate positive correlation. The $p$-value was $<.00001$. The result is significant at $p<.05$. Further studies must be carried on with large sample size and involving different ethnic group. We are very grateful to all participated women whom voluntarily accept to engage in the study and to all cooperative staff at banoon fertility center.
\end{abstract}

Key Words: Antimullerian hormone, laparoscopy, ovaries, platelets rich plasma

Received: 22 October 2019, Accepted: 23 November 2019

Corresponding Author: Mosab Nouraldein Mohammed Hamad, Banoon Fertility Center, Khartoum, Sudan, Tel.: +249929194137, E-mail: musab.noor13@gmail.com

ISSN: 2090-7625, February 2020, Vol.10, No.1

\section{INTRODUCTION}

Platelet-rich plasma is known as (a quantity of plasma that has a platelet count over baseline $)^{[1]}$. Platelets are cytoplasmic remains of megakaryocytes; produced in the bone marrow ${ }^{[2,3]}$. The woman ovary enclosed with an epithelial monolayer which preserves cyclic ‘injury' and restricted tissue repair with every ovulation. Whereas inhabitant stem cells have thought fundamental for the recovery essential for hemostasis and organ ruling here, the behavior and mode of activity for these cells remains not entirely characterized. Indeed in spite of the fact that later ask about has opened an entrance into ovarian stem cell science $^{[4,5]}$. Though platelets play a key role in hemostasis, they are central to mediating anabolic consequences of PRP by virtue of discharging growth factors stored in their alpha granules. Throughout the first phases of wound repair, activated platelets attract and promote cell migration into the wound by aggregating and forming a fibrin matrix. This matrix then serves as a tissue scaffold for sustained free of platelets growth factors and cytokines, which stimulate cell recruitment, differentiation and communication ${ }^{[6]}$.

A tendency of reduced pregnancy rate observed in women with thin endometrium when contrasted with women with thick endometrium. Thin endometrium much raises the risk of embryo implantation failure ${ }^{[7]}$.

Many efforts to enhance the thickness of endometrium, several approaches have made. Platelets hold a significant measure of growth factors that have positive effects on local tissue repair ${ }^{[8,9]}$. Larson et al. productively developed bovine embryos through the fourth cell cycle using platelet derived growth factor (PDGF) ${ }^{[10]}$. This was the beginning of an evolution in decreasing abortion rate by raising endometrial thickness. Chang et al. directed intrauterine infusion of platelet rich plasma (PRP) in infertile women with thin endometrium and accounted good results four pregnancies from five patients with thin endometrium and poor response to conventional therapy through freeze embryo transfer ${ }^{[1]}$.

Since of a central issue in many clinical infertility introductions are ovarian senescence and an unavoidable decay in oocyst donation, it appears sensible to consider utilizing autologous PRP in a reproductive setting. Basically since the thought of diminished (or totally lost) fertility potential related with ovarian failure is the center of ongoing investigate, the prospect of PRP progressing the ovarian microenvironment- and indeed collaboration 
with putative ovarian germline stem cells (GSCs)- call for genuine consideration.

\section{Rationale:}

In spite of its extensive applications in medicine, the methods through which PRP exerts its regenerative effects are only hypothesized, not stand on enough scientific records. Then we want to enrich that scientific data about uses of PRP as therapeutic tool for women's subfertility.

\section{Objectives:}

General objectives: To know the size of correlation between laparoscopic ovarian autologous PRP injection and $\mathrm{AMH}$ level.

Specific objectives: To evaluate significance of laparoscopic ovarian autologous PRP on AMH level.

\section{PATIENTS AND METHODS}

Study design: Randomized clinical trial

Study setting: Banoon fertility center, Khartoum, Sudan

Study period : January 2018- June 2019

Study population: Subfertile women attended to Banoon fertility center and exposed to Laparoscopic ovarian PRP during the study period.

\section{Inclusion criteria:}

- Subfertile woman with normal blood pressure

- Subfertile woman free from heart disease

- Not used Aspirin.

- Normal bleeding profile

- AMH level below $0.5 \mathrm{ng} / \mathrm{ml}$

\section{Exclusion criteria:}

- Hypertensive patients.

- Cardiac disease patients.

- Aspirin users.

- Patients with abnormal bleeding profile.

- AMH level above $0.5 \mathrm{ng} / \mathrm{ml}$

Sample size : 51 subfertile women, whom represented all number of women examined for AMH level before and after injection with laparoscopic ovarian aPRP .

Ethical approval: The study approved by Sudanese national research ethical committee and research ethical committee in Banoon center for infertility, all participants informed about the aims of the study and they were consent to involved in it.

Statistical analysis: Microsoft Excel and SPSS statistical methods.

\section{Measurement of $A M H$ :}

VIDAS AMH (AMH) is an automated test for use on the VIDAS family of instruments, for the quantitative measurement of circulating anti-Müllerian Hormone (AMH) in human serum or plasma (lithium heparin) the usage of the ELFA (Enzyme Linked Fluorescent Assay) method.

The measure guideline joined a one-step enzyme immunoassay sandwich technique with a last fluorescent detection (ELFA)

The Solid Phase Receptacle (SPR) served as the solid phase as well as the pipetting device. Reagents for the assay were ready-to-use and pre-dispensed in the sealed reagent strips.

All of the measure steps performed habitually by the instrument. The response medium cycled in and out of the SPR a few times.

The specimen exchanged into the wells containing antiMüllerian antibody labeled with alkaline phosphatase (conjugate). The sample/conjugate mixture cycled in and out of the SPR several times. This operation empowers the anti-Müllerian hormone to bind with the antibodies coated on the insides of the SPR and with the conjugate to create a sandwich. Unbound components eradicated during the washing steps.

Throughout the last detection step, the substrate (4-Methylumbelliferyl phosphate) cycled in and out of the SPR. The conjugate enzyme catalyzes the hydrolysis of the substrate into a fluorescent product (4-Methylumbelliferone), fluorescence of which estimated at $450 \mathrm{~nm}$. Escalated of the fluorescence was corresponding to the concentration present in the sample.

At conclusion of the test, the results were automatically calculated by the device to the calibration curve stored in memory, and after that printed out.

Antimullerian hormone level measured twice, before injection of autologous PRP to the ovaries and after 3 months of its injection to ovaries.

Sample collection and preparation of PRP: All blood specimens collected under aseptic condition. 40 $\mathrm{ml}$ blood collected from each participant by peripheral venipuncture using a $21 \mathrm{G}$ butterfly catheter affixed via vacutainer to negative pressure receiving tubes. Samples were immediately labeled and placed in roomtemperature centrifuge first for 15 minutes at 1300RPM (Figure 1), got poor platelet plasma (Figure 2) and then for 8 minutes at 3300RPM (Figure 3), obtained platelet rich plasma (Figure 4), then $0.08 \mathrm{ml}$ of $10 \% \mathrm{w} / \mathrm{v} \mathrm{Ca}++$ gluconate added 
as activator to every $3 \mathrm{ml}$ of PRP (Figure 5) and after 10 minutes two $5 \mathrm{ml}$ syringe filled with $2.5 \mathrm{ml}$ of activated PRP administered into each ovary with laparoscopic

Images to the steps of laparoscopic ovarian PRP injection:

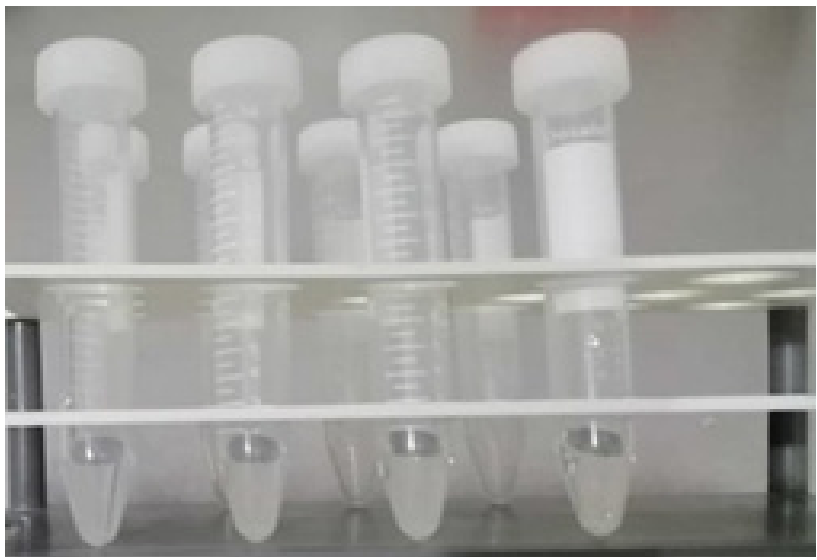

Fig.1: Centrifugation of blood at 1300rpm for 15 minutes

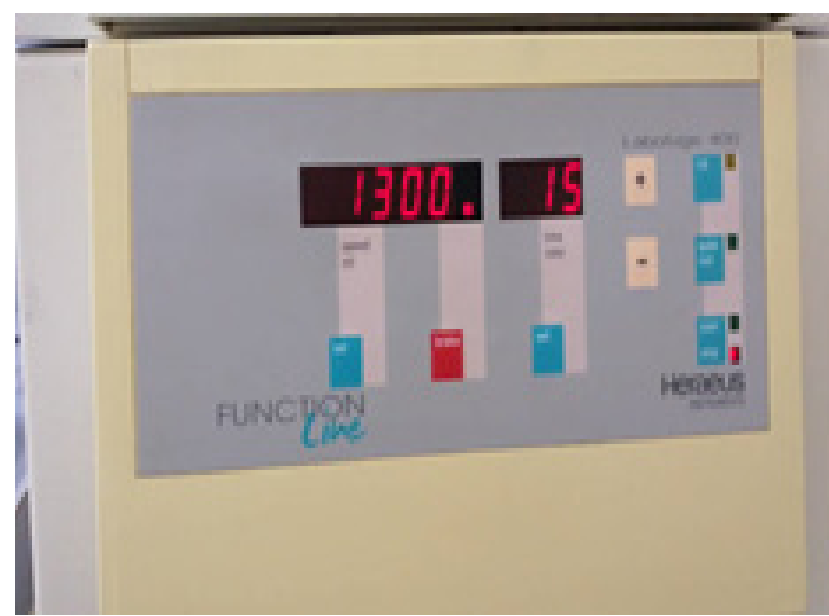

Fig.3: Centrifugation of platelets poor plasma at 3300rpm for 8

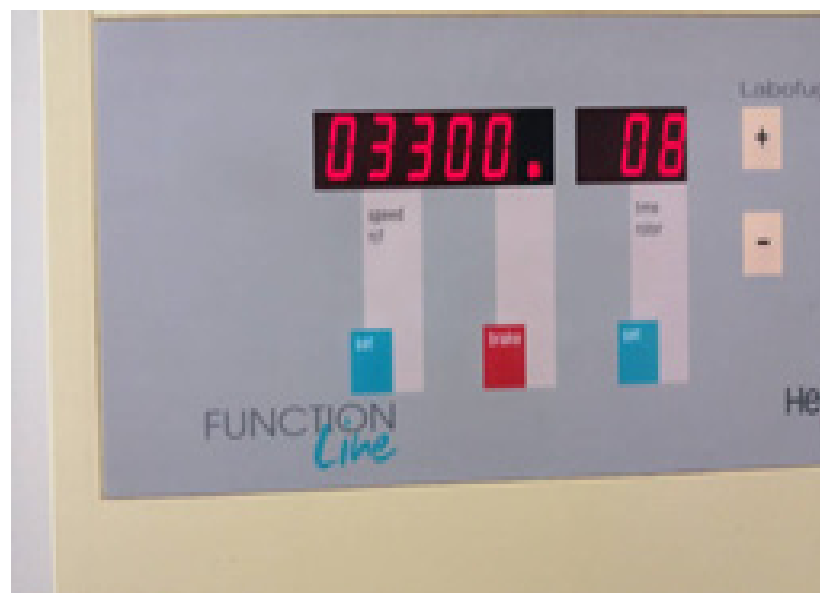

Fig.5: Two sterile syringes contain activated PRP guidance (Figures 6, 7). Mini vidas used to measure AMH level before and after 3 months of autologous PRP injection to the ovaries (Figure 8).

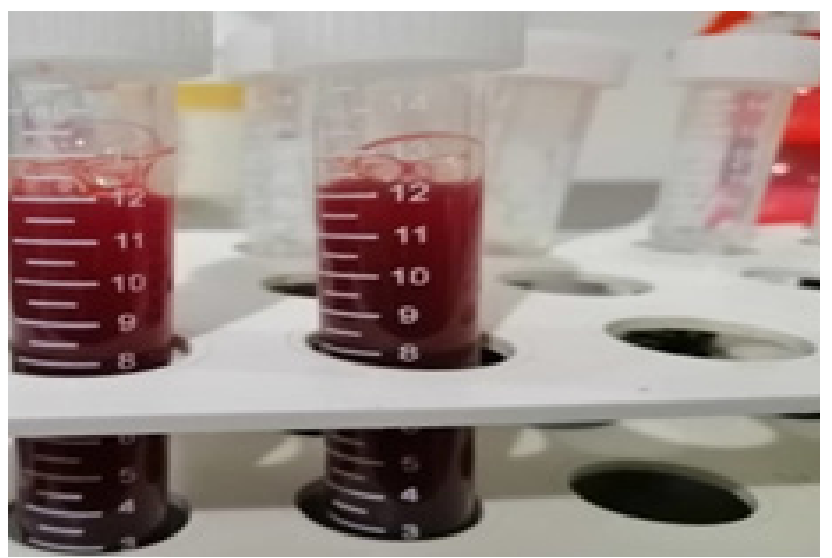

Fig.2: Poor platelets plasma

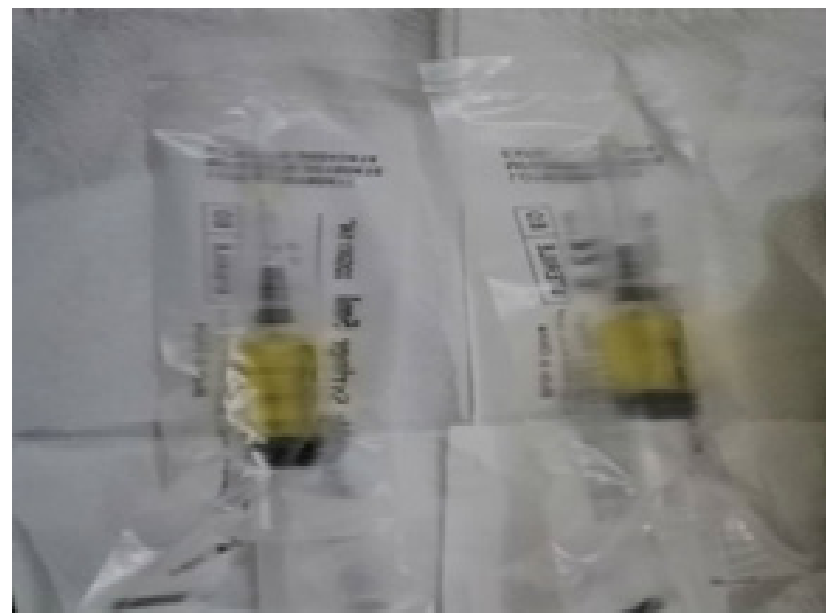

Fig. 4: Platelets rich plasma

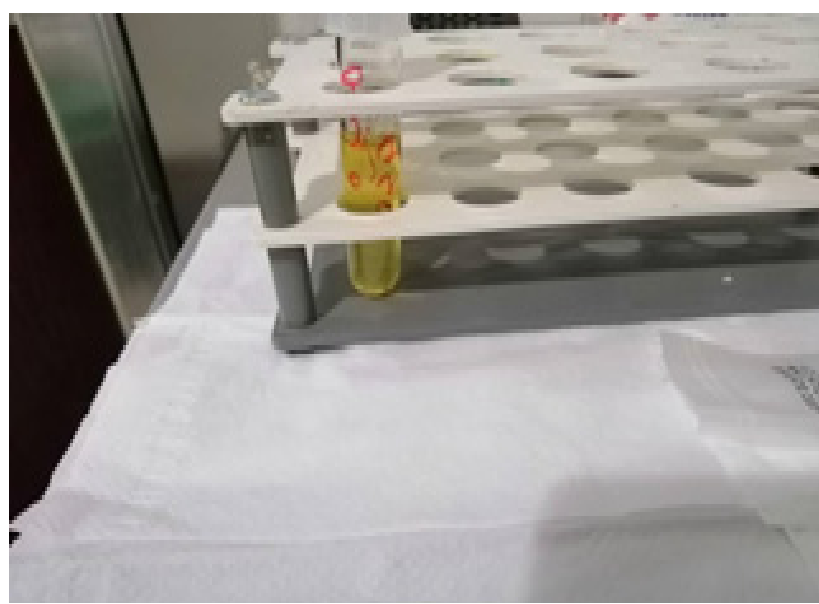

Fig. 6: Injection of PRP to the right ovary 


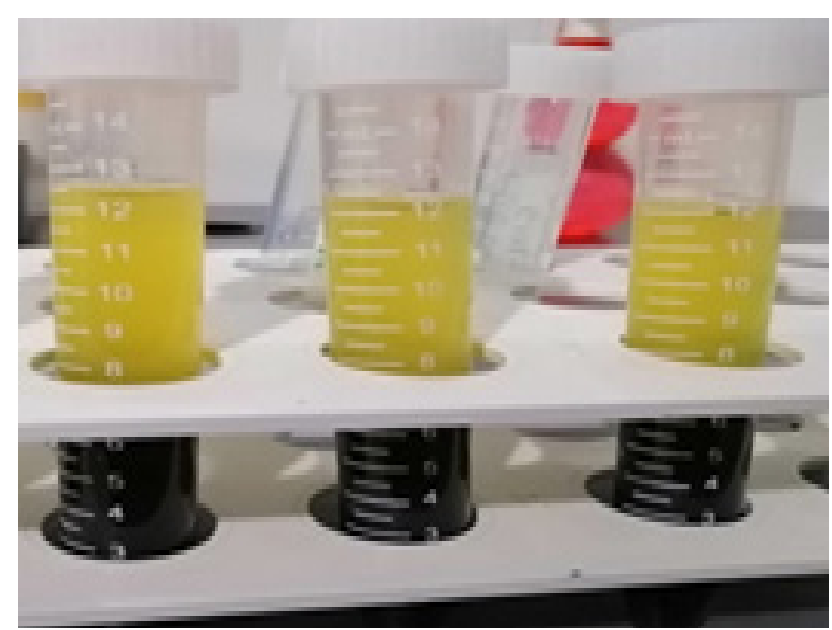

Fig.7: Injection of PRP to the left ovary

\section{RESULTS}

AMH levels reading before and after PRP were increased in $33.3 \%$ (17), not changed in $37.3 \%$ (19) and decreased in $29.4 \%$ (15) (Table 1). Pregnancy rate was $7.84 \%$ (4) (Table 2) and 23.5\% (4) (Table 3) from

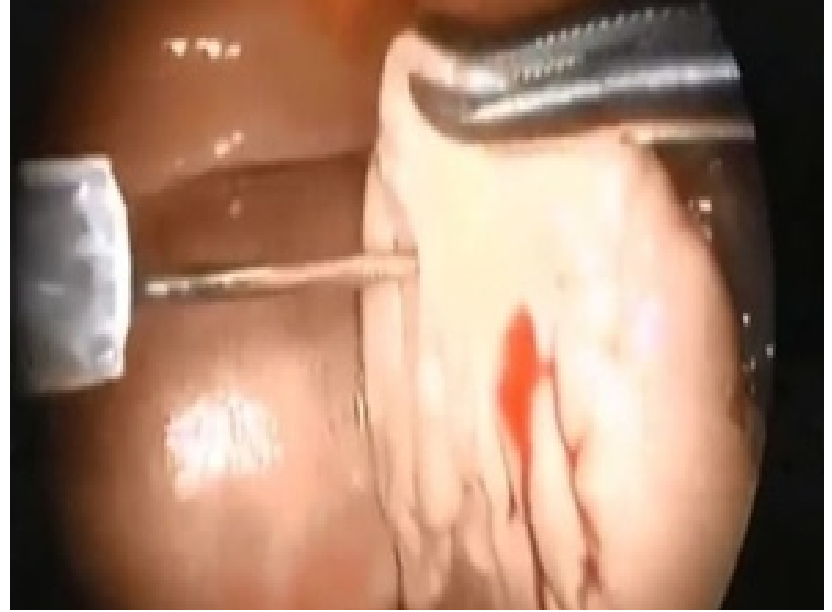

Fig. 8 : Mini Vidas used for AMH measurement

the total participants and those with increased AMH level, respectively, one got pregnant with twins and 3 with single baby. The size of correlation equals 0.64 which represent moderate positive correlation (Figure 9). The $p$-value was $<.00001$. The result was significant because $p<.05$.

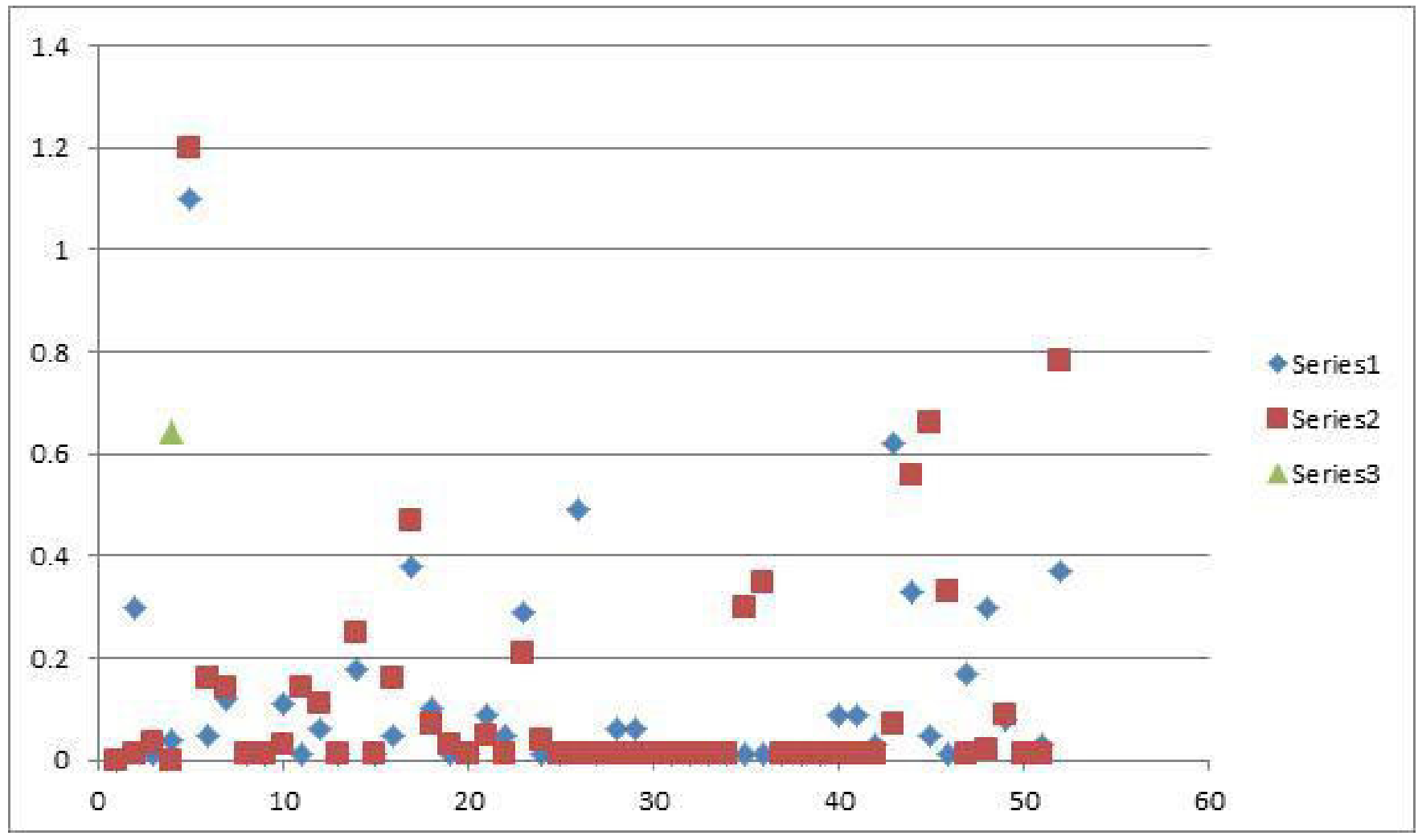

Fig.9: Correlation between AMH level before and after PRP injection

Series 1: AMH before PRP injection

Series 2: AMH before PRP injection

Series 3: Correlation level (0.64). 
Table 1: Showed level of AMH before and after injection of autologous PRP to ovaries

\begin{tabular}{lcc}
\hline Criteria & Number & Percentage \\
\hline participant with increased AMH & 17 & $33.3 \%$ \\
Participants with constant AMH & 19 & $37.3 \%$ \\
Participants with decreased AMH & 15 & $29.4 \%$ \\
Total & 51 & $100 \%$ \\
\hline
\end{tabular}

Table 2: Showed pregnancy rate among the total participants

\begin{tabular}{lcc}
\hline Criteria & Number & Percentage \\
\hline $\begin{array}{l}\text { Got pregnant after exposure to ovarian aPRP } \\
\text { injections }\end{array}$ & 4 & $7.84 \%$ \\
$\begin{array}{l}\text { Not pregnant after exposure to ovarian aPRP } \\
\text { injection }\end{array}$ & 47 \\
Total & 51 & $92.16 \%$ \\
\hline
\end{tabular}

Table 3 : Showed pregnancy rate among the participants with increased AMH after exposure to ovarian aPRP injections

\begin{tabular}{lcc}
\hline Criteria & Number & Percentage \\
\hline $\begin{array}{l}\text { Got pregnant after exposure to ovarian aPRP } \\
\text { injections }\end{array}$ & 4 & $23.5 \%$ \\
$\begin{array}{l}\text { Not pregnant after exposure to ovarian aPRP } \\
\text { injection }\end{array}$ & 13 & $76.5 \%$ \\
Total & 17 & $100 \%$ \\
\hline
\end{tabular}

\section{DISCUSSION}

The present study showed that PRP had much positive effect on AMH level which means that laparoscopic injection of PRP to the ovaries can be regarded as good rejuvenation tool to the ovaries of subfertile women, it is supported by the outcomes of the study done by Abdullah Thuraya Husamuldeen et al. that showed a significant improvement of instead of ovarian reserve tests after PRP injection and increase in AMH level ${ }^{[12]}$. Due to small sample size and restricted ethnic group we cannot judge that this method is effective to raise level of AMH to every subfertile female, but it can be taken as a factor that may enhance opportunities of improved.

\section{CONCLUSION}

Our study concluded that PRP is an active rejuvenation method to ovaries of subfertile women. Further studies should be done with large sample size participants and involve different ethnic groups.

\section{CONFLICT OF INTEREST}

There are no conflicts of interests.

\section{REFERENCES}

1. Zhu Y, Yuan M, Meng HY, Wang AY, Guo $\mathrm{QY}$, Wang $\mathrm{Y}$, et al. Basic science and clinical 
application of platelet-rich plasma for cartilage defects and osteoarthritis:a review. Osteoarthritis Cartilag. 2013;2:1627-37.

2. Conley CL. Hemostasis. In: Mountcastle VB, editor. Medical Physi-ology. St. Louis: The C.V. Mosby Company; 2004. p. 1137-46.

3. Harrison P, Cramer EM. Platelet alpha-granules. Blood Rev 1993;7:52-62

4. Tilly JL, Sinclair DA. Germline energetics, aging, and female infertility. Cell Metab 2013;17:838-50.

5. Ng A, Barker N. Ovary and fimbrial stem cells: biology, niche and cancer origins. Nat Rev Mol Cell Biol 2016;16:625-38.

6. Scherer SS, Tobalem M, Vigato E, et al. Nonactivated versus thrombin -activated platelets on wound healing and fibroblast-to-myofibroblast differentiation in vivo and in vitro. Plast Reconstr Surg 2012;129(1):46e-54e.

7. Casper RF. It's time to pay attention to the endometrium. Fertil Steril 2011;96:519-21.
8. Macaulay IC, Carr P, Gusnanto A, Ouwehand WH, Fitzgerald D, Watkins NA. Platelet genomics and proteomics in human health and disease. J Clin Invest. 2005;115(12):3370-3377.

9. Rudkin GH, Miller TA. Growth factors in surgery. Plast Reconstr Surg. 1996;97(2):469-476.

10. Larson R, Ignotz G, Currie W. Platelet derived growth factor (PDGF) stimulates development of bovine embryos during the fourth cell cycle. Development. 1992;115(3):821-826.

11. Chang $\mathrm{Y}$, Li J, Chen $\mathrm{Y}$, Wei L, Yang $\mathrm{X}$, Shi Y, Liang X. Autologous platelet-rich plasma promotes endometrial growth and improves pregnancy outcome during in vitro fertilization. Int $\mathrm{J}$ Clin Exp Med. 2015;8(1):1286-1290.

12. Abdullah Thuraya Husamuldeen, Abbas Sallama Hamid, Al-Obaidi Manal Taha, Abdulraheem Yousif, The Efficacy of Platelets Rich Plasma (PRP) for Ovarian Rejuvenation. Indian Journal of Public Health Research \& Development, 2019, Volume: 10, Issue 8,p: 1211-1217 